\title{
EIEMENT ABUNDANCES OF NOVA PW VULPECULAE
}

\author{
J. Andreae and $\mathrm{H}$. Drechsel \\ Dr. Remeis-Observatory Bamberg \\ Astronomical Institute of the University Erlangen Nuemberg. F.R.G.
}

\begin{abstract}
Element abundances, electron temperatures and densities of the shell ejected during the outburst of the classical nova PW Vulpeculae were determined using ultraviolet (UUE) and optical ESO 1.52m + B\&C + IDS) spectra obtained during the nebular phase (April - July 1985). The C. N. O abundances are enhanced by factor 10 - 100 relative to solar values according to the predictions of the TNR (thermonuclear runaway) theory. The overabundances of $\mathrm{Ne}, \mathrm{Si}, \mathrm{Mg}$ are comparatively small compared with other novae indicating that the outburst of this slow nova occurred on a $\mathrm{CO}$ white dwarf.
\end{abstract}

\section{Electron Temperatures and Densities}

A set of 17 quasi-simultaneous IUE and optical spectra which were obtained $340 \pm 15$ days after maximum brightness during the nebular phase were used for the present analysis.

Electron temperatures can be determined using the flux ratios of lines produced by dielectronic recombination relative to those produced by collisional excitation (see e.g. Williams et al.,

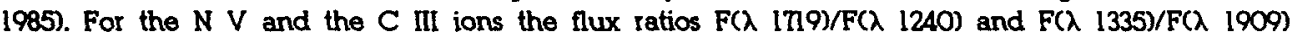
were used, respectively. A color excess of $E(B-V)=0.50$ was used, which could be determined with the aid of the $2200 \AA$ feature and the He II recombination theory. The results for the electron temperatures are

$$
T_{0}(N)=14700 \pm 800 \mathrm{~K} \text { and } T_{e}(C \text { IID }-10100 \pm 600 \mathrm{~K} \text {. }
$$

The electron density can be determined using $O$ III ratios of UV and optical emission line fluxes

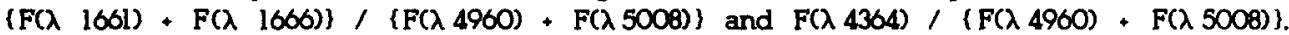
From the diagnostic diagram which was calculated using a computer code of Snijders (1989) we obtain densities of $\log \left(\mathrm{N}_{\mathrm{e}}\right) \cdot 0.9$ and $\log \left(\mathrm{N}_{\mathrm{e}}\right)$ - 6.7. respectively, if we interpolate the electron temperature of $O$ III as a function of the ionization potential. We adopt

$$
\log \left(\mathrm{N}_{\mathrm{e}}\right)=7.0 \pm 0.5\left(\mathrm{~cm}^{-3}\right)
$$

as value for the electron density.

\section{Element Abundances}

We have calculated ion abundances for the beginning of July 1985, using UV line fluxes of collisionally excited lines obtained on June 24 and optical line fluxes obtained on July 1,2 and 6. We interpolate the electron temperature as a function of ionization potential and adopt the value $\log \left(\mathrm{N}_{e}\right)=7.0$ for the electron density.

The $\mathrm{H} \mathrm{I}, \mathrm{He} \mathrm{I}$ and $\mathrm{He}$ II lines are produced by recombination Catomic data were e.g. calculated by Hummer and Storey, 1987), and we obtain abundance ratios $\mathrm{N}$ (He II) / N OH ID $=0.080$ using $\mathrm{He}$ I $\lambda 5876$ and $\mathrm{H} \alpha$, estimating a contribution of $10 \% \mathrm{~N}$ II $(\lambda 6548+\lambda 6583)$ to the $\mathrm{H} \alpha$ feature, and $N$ Ge IID / N CH II) - 0.04l, using He II $\lambda 4686$ and $H \beta$.

Applying an ionization correction factor (c.f. Snijders et al., 1980 for unobserved ionization stages, assuming that only $10 \%$ of the total amount of $\mathrm{N}$ is not observed, we get values for the element abundances relative to $\mathrm{H}$ : 


\begin{tabular}{c|ccccccccc} 
Element & $\mathrm{H}$ & $\mathrm{He}$ & $\mathrm{C}$ & $\mathrm{N}$ & $\mathrm{O}$ & $\mathrm{Ne}$ & $\mathrm{Mg}$ & $\mathrm{Si}$ & $\mathrm{Fe}$ \\
\hline N(Element) / N (H) & 1.00 & 0.12 & 0.011 & 0.017 & 0.012 & $2.0 \theta-4$ & $2.3 \theta-5$ & $4.3 \theta-4$ & $3.4 \theta-5$
\end{tabular}

Finally. the mass fractions of the elements in the shell ejected by nova PW Vulpeculae are calculated and shown together with the results for other novae in the table below. For comparison, solar values are included. "SC" gives the nova speed class ( $v f$ - very fast, $\mathrm{mf}$ - moderate fast, s - slow). Most values are from Truran 0985).

The visual lightcurve of nova PW Vulpeculae shows quasiperiodic oscillations just after maximum light. The characteristic times for the decline by two and three magnitudes were determined by smoothing these oscillations. We obtain $t_{2}-83 \pm 4$ days and $t_{3}=147 \pm 6$ days indicating that nova PW Vulpeculae belongs to the slow novae.

\begin{tabular}{l|cccccccc} 
Object & SC & H & He & C & N & O & Ne & Z \\
\hline Sun & - & 0.74 & 0.24 & 0.0039 & 0.0094 & 0.0088 & 0.0021 & 0.019 \\
RR Pic & $\mathrm{s}$ & 0.53 & 0.43 & & 0.022 & 0.0058 & 0.011 & 0.0039 \\
HR Del & $\mathrm{s}$ & 0.45 & 0.48 & & 0.027 & 0.047 & 0.003 & 0.077 \\
T Aur & $\mathrm{s}$ & 0.47 & 0.40 & & 0.079 & 0.051 & & 0.13 \\
Mus 1983 & $\mathrm{mf}$ & 0.43 & 0.38 & 0.0004 & 0.12 & 0.07 & & 0.20 \\
PW Vul & $\mathrm{s}$ & 0.49 & 0.23 & 0.064 & 0.12 & 0.093 & 0.0019 & 0.28 \\
Cyg 1975 & $\mathrm{vf}$ & 0.49 & 0.21 & 0.070 & 0.075 & 0.13 & 0.023 & 0.30 \\
Cyg 1978 & $\mathrm{mf}$ & 0.45 & 0.23 & 0.047 & 0.14 & 0.13 & 0.0068 & 0.32 \\
CrA 1981 & $\mathrm{mf}$ & 0.31 & 0.31 & 0.0046 & 0.080 & 0.12 & $0.1 \mathrm{~T}$ & 0.38 \\
DO Her & $\mathrm{s}$ & 0.34 & 0.095 & 0.045 & 0.23 & 0.29 & & 0.56 \\
Aql 1982 & vf & 0.01 & 0.02 & 0.18 & 0.03 & 0.40 & 0.15 & 0.97
\end{tabular}

The table shows that the $\mathrm{Z}$ value for nova PW Vulpeculae is intermediate among the values determined so far. The results for the element abundances fit the predictions of the TNR theory and are in the same range like those of other classical novae. It should be stressed that especially the abundances for the slow nova PW Vulpeculae resemble closely the values of the very fast nova Cygni 1975, though these two objects are of completely different speed class.

Presently we are determining element abundances for other novae in order to extend the set of abundances for novae, and for some symbiotic stars.

\section{References}

Hummer. D. S.. Storey. P. J.: 1987. Monthly Notices Roy. Astron. Soc. 204 801

Osterbrock. D. E.: 1974. Astrophysics of Geseous Nebulae, Freeman, San Francisco

Snijders. M. A. J.: 1989, priv. comm.

Snijders. M. A. J., Batt, T. J., Roche, P. F., Seaton, M. J., Morton, D. C., Spoelstra, T. A. T., Blades, J. C.: 1981. Monthly Notices Roy. Astron. Soc. 208, 329

Stickland, D. J., Penn, C. J.. Seaton, M. J., Snijders, M. A. J., Storey, P. J.: 1981. Monthly Notices Roy. Astron. Soc. 191. 107

Truran, J. W.: 1985, Proc. of the ESO Workshop on "Production and Distribution of the C. N, O Elements", Garching. 211

Williarns, R. E. Ney, E. P., Sparks, W. M., Starrield, S. G., Wyckoff, S., Truran, J. W.: 1985, Monthly Notices Roy: Astron. Soc. 22, 753 